\title{
Respuesta del consumidor y su relación con dos características sensoriales de una bebida
}

\section{Consumer response and its relationship with two sensorial characteristics of a beverage}

\author{
Eliana G. Contreras-López ${ }^{1}$, Ricardo Yuli Posadas ${ }^{1}$, María A. Arosena ${ }^{2}$, Ruth H. Chavez ${ }^{2}$, Elizabeth M. Palacios ${ }^{2}$, Sofía V. Huamán ${ }^{2}$ \\ ${ }^{1}$ Universidad Nacional Mayor de San Marcos. Lima, Perú. \\ ${ }^{2}$ Universidad Nacional Mayor de San Marcos, Facultad de Farmacia y Bioquímica. Lima, Perú.
}

\section{Resumen}

El objetivo de esta investigación fue determinar la respuesta del consumidor respecto al nivel de agrado de una bebida en relación a las características sensoriales de sabor y consistencia. La bebida fue elaborada en base a pulpa de piña (Ananas comosus) y quinua (Chenopodium quinoa Willd), en proporción 7:1 (p:p), procesada mediante tres tratamientos térmicos diferentes TP01, TP02 y TP03 $\left(85^{\circ} \mathrm{C} \times 26\right.$ minutos, $90^{\circ} \mathrm{C} \times 7$ minutos y $95^{\circ} \mathrm{C} \times 2$ minutos). Las características físico-químicas evaluadas fueron: $\mathrm{pH}$, grados Brix y viscosidad. Los atributos sensoriales de consistencia y sabor (global) fueron evaluados con un panel conformado por 15 jueces entrenados. Los resultados presentaron diferencias estadísticamente significativas entre los tratamientos en cuanto a la viscosidad y consistencia; en la evaluación de sabor como atributo sensorial global no se reportaron diferencias entre los tres tratamientos. El nivel de agrado del consumidor se llevó a cabo con personas entre 16 y 40 años de edad; quienes no encontraron diferencias significativas respecto al nivel de agrado de la bebida. Se concluye que el atributo sensorial de consistencia no fue determinante en la preferencia del consumidor, pudiendo existir correlación positiva en la preferencia del consumidor con el sabor. La preferencia por la bebida es mayor en los consumidores del género femenino y los consumidores más jóvenes (16 - 18 años de edad).

Palabras claves: Análisis sensorial; bebida de piña (Ananas comosus) y quinua (Chenopodium quinoa Willd); nivel de agrado del consumidor.

\section{Abstract}

The objective of this research was to determinate the consumer response about the degree liking of a beverage in function to flavor and consistency. The beverage was elaborated with pineapple (Ananas comosus) pulp/quinoa (Chenopodium quinoa Willd), in ratio 7:1 (w:w), processed in three heat treatments TP01, TP02 and TP03 $\left(85^{\circ} \mathrm{C} \times 26\right.$ minutes, $90{ }^{\circ} \mathrm{C} x$ 7 minutes and $95^{\circ} \mathrm{C} \times 2$ minutes). The physical-chemical characteristics evaluated were: $\mathrm{pH}$, degrees Brix, viscosity. The sensory evaluation was evaluating with trained judges (panel of 15 judges) to flavor and consistency. The results showed statistically significant differences between the three treatments in terms of viscosity and consistency; in flavor not showed statistically significant differences between treatments. The linking degree for consumers employed people of 16 to 40 years old. The consumer's response not present significant differences. In conclusion, the consistency no was determinate in the consumer preference. Could be a correlation between the consumer's preference and flavor. The female consumers and the younger consumers prefer the beverage.

Key words: Sensory analysis; pineapple (Ananas comosus) pulp; quinoa (Chenopodium quinoa Willd); beverage; consumer preference.

\section{Correspondencia: \\ Nombre: Eliana Gabriela Contreras López \\ Dirección: Jr. Puno 1002. Lima, Perú.}

Correo: econtrerası@unmsm.edu.pe

Recibido: 15/12/2017

\section{Citar como:}

Contreras-López, E., Yuli, R., Arosena, M., Chavez, R., Palacios, E., Huamán, S. Respuesta del consumidor y su relación con dos características sensoriales de una bebida. Ciencia e Investigación 2018 21(1):49-54.

(c) Los autores. Este artículo es publicado por la Ciencia e Investigación de la Facultad de Farmacia y Bioquímica de la Universidad Nacional Mayor de San Marcos. Este es un artículo de acceso abierto, distribuido bajo los términos de la licencia Creative Commons Atribucion - No Comercia_Compartir Igual 4.0 Internacional. (http://creativecommons.org/licenses/by-nc-sa/4.0/) que permite el uso no comercial, distribución y reproducción en cualquier medio, siempre que la obra original sea debidamente citada. 


\section{INTRODUCCIÓN}

En los últimos años el consumidor es cada vez más exigente sobre la calidad de los alimentos que obtiene demandando alimentos saludables, inocuos y de alta calidad sensorial; representando un desafío para la industria alimentaria ${ }^{1}$. Por tanto, se hace imprescindible hacer un estudio para conocer la respuesta de los consumidores respecto a sus gustos, aversiones y preferencias a fin de desarrollar nuevos alimentos o mejorar la calidad de los existentes $^{2,3}$.

En la ciudad de Lima se ofrecen en puestos ambulantes bebidas a base de piña (Ananas comosus) y quinua (Chenopodium quinoa Willd.), las cuales son aceptadas por su sabor agradable y consumidas por su bajo costo y rapidez con la que se sirven ${ }^{4}$, principalmente en horas de la mañana como parte del desayuno, sin embargo este tipo comercialización en la vía pública representa un riesgo a los consumidores, por la posible carencia de controles sanitarios, que no ofrecen garantías higiénicas ni sanitarias.

En la elaboración de esta bebida se emplean básicamente dos materias primas, que al procesarse, se obtiene un producto muy agradable. La piña es el fruto de la familia de las bromeliáceas proporciona varios minerales esenciales, vitaminas $\left(\mathrm{B}_{1}, \mathrm{~B}_{2}, \mathrm{C}\right)$, rico en carbohidratos, pobre en lípidos y proteínas ${ }^{5,6}$, cuyo sabor está en función del contenido de azúcares totales, los cuales varían de acuerdo al clima y el grado de madurez de la cosecha 7. La variedad denominada Golden Sweet, se caracteriza por la baja acidez y altos contenidos de sólidos solubles totales (14-18 ${ }^{\circ}$ Brix en promedio, pudiendo alcanzar en condiciones excepcionales hasta $30^{\circ}$ Brix. ${ }^{5}$ ). La quinua es una excelente materia prima para la elaboración de los denominados alimentos saludables; la quinua es un pseudocereal originario de la región andina de América del Sur, cuyo grano es altamente nutritivo debido a su excelente calidad proteica, perfil equilibrado de aminoácidos, amplia gama de minerales y vitaminas. ${ }^{8,9}$ En relación con otros granos dietéticos como el trigo, arroz, maíz, avena, y cebada, ésta tiene un contenido más alto de proteínas. ${ }^{10}$ Se considera de fácil digestión porque no contiene gluten y es buena fuente de proteínas (12-18 $\mathrm{g} / 100 \mathrm{~g}$ peso seco), fibra, fósforo, magnesio y hierro. Además, contiene una gran variedad de compuestos antioxidantes, tales como carotenoides, vitamina $\mathrm{C}$ y flavonoides, que son protectores contra una variedad de enfermedades, como cáncer, alergias y reducen el riesgo de enfermedades cardiovasculares. No obstante, la quinua contiene componentes de sabor amargo (principalmente saponinas hidrosolubles) que se eliminan con el lavado antes del consumo ${ }^{11}$.

En la elaboración de bebidas es importante conocer el tratamiento térmico que se le dará a fin de garantizar la inocuidad y asimismo alargar la vida útil. Las bebidas a base de frutas cuyos valores de $\mathrm{pH}$ están en el rango de 4,0 - 3,7, son consideradas dentro del grupo de alimentos muy ácidos ${ }^{12}$, por tanto, el tratamiento térmico adecuado para este tipo de alimentos a aplicar es la pasteurización y se considera al Bysosschlamys fulva como el microorganismo de referencia ${ }^{13}$. Tradicionalmente la pasteurización por calentamiento discontinuo se da en el rango de temperatura desde los $60^{\circ} \mathrm{C}$ hasta los $85^{\circ} \mathrm{C}$ por tiempos comprendidos entre 7 a 15 minutos ${ }^{5}$. La pasteurización puede causar modificaciones tanto en la calidad nutricional ${ }^{14}$ como en la calidad sensorial ${ }^{5}$.

En la elaboración de bebidas representa un desafío la preservación la calidad sensorial ${ }^{15}$, los cuales son valorados por el consumidor antes de elegir un alimento. En general una actitud favorable hacia un producto puede significar que el consumidor tiene una mayor propensión a comprarlo ${ }^{16}$. En este contexto se estableció como objetivo principal de la investigación determinar la respuesta del consumidor (nivel de agrado) de una bebida elaborada a base de pulpa de pińa y quinua en relación a las características sensoriales de sabor y consistencia, bajo la hipótesis que el tratamiento térmico de la bebida afecta a los atributos sensoriales de sabor (global) y consistencia.

\section{MATERIAL Y MÉTODOS}

\section{Elaboración de la bebida}

Se seleccionaron las frutas (piña de la variedad golden sweet) procedentes de la provincia de Satipo, departamento de Junín, sin daños físicos, químicos o microbiológicos. Se lavaron en agua corriente y se sumergieron en agua clorada (100 ppm por 10 minutos), luego se pelaron manualmente y trozaron en cubos de aproximadamente $2,5 \mathrm{~cm}$. Se escaldaron a $82^{\circ} \mathrm{C}$ por dos minutos $\mathrm{y}$ se trituraron en un procesador de frutas, se tamizó para tener la pulpa refinada que se almacenó en congelación hasta el mezclado.

La quinua utilizada fue la blanca de la variedad Salcedo INIA, procedente de la provincia de Huamanga, departamento de Ayacucho, fue seleccionada, lavada dos veces con agua corriente y sometida a cocción a $100^{\circ} \mathrm{C}$ hasta que cada grano dobló su volumen y su germen espiral se separó, luego se trituró en una licuadora. Posteriormente se mezcló la pulpa de pińa con la quinua triturada en proporción 7:1 (p:p), se agregó azúcar granulada (sacarosa), se pasteurizó según los parámetros mostrados en la Tabla 1. Finalmente se envasó en caliente en botellas de vidrio con tapa metálica y luego se sumergió en un baño de agua mantenida a temperatura ambiente $\left(22^{\circ} \mathrm{C}\right)$.

\section{Tratamiento térmico:}

La determinación del tiempo de tratamiento térmico, se calculó usando la siguiente ecuación:

$$
D=D_{0} \times 10^{\frac{1}{z}\left(T_{0}-T\right)}
$$

El tiempo de tratamiento térmico necesario para reducir la población inicial de microorganismos en $3 \mathrm{D}$ con las temperaturas de pasteurización: $85^{\circ} \mathrm{C}, 90^{\circ} \mathrm{C}$ y $95^{\circ} \mathrm{C}$, se muestran en la Tabla 1. Los parámetros base del microorganismo de referencia (Bysosschlamys fulva) fueron $\mathrm{D}_{93,3^{\circ} \mathrm{C}}=1,0$ minuto y valor $\mathrm{Z}=8,9^{\circ} \mathrm{C}$ y la temperatura mínima letal $=70^{\circ} \mathrm{C}^{13}$. 


\section{Análisis fisicoquímico}

Las propiedades fisicoquímicas determinadas fueron $\mathrm{pH}$, ${ }^{\circ}$ Brix y viscosidad. El $\mathrm{pH}$ se midió usando un potenciómetro de mesa Accumet, el contenido de sólidos solubles fue medido utilizando un refractómetro (Marca: Milwaukee modelo MA871, 0- $85^{\circ}$ Brix / $0-80^{\circ} \mathrm{C}$ ) de precisión $\pm 0.2 \mathrm{Brix} / \pm 0.3^{\circ} \mathrm{C}$. La viscosidad se midió con un viscosímetro modelo RVDV-E - Brookfield, a una velocidad de $100 \mathrm{rpm}$, empleando el spindle (SS) $\mathrm{N}^{\circ} 00$, puesto que este tipo de spindle se recomienda para liquidos poco viscosos con una medición de torque de $1 \%$, la muestra se midió en un vaso de precipitado de $500 \mathrm{~mL}$.

\section{Análisis sensorial de la bebida de quinua y pińa}

Evaluación sensorial y panel de jueces entrenados

Según la NTP-ISO 5492 (2008) ${ }^{17}$, juez entrenado es el catador con un grado elevado de sensibilidad sensorial y experiencia en su metodología, el cual es capaz de efectuar un juicio fiable de diversos productos por medio del análisis sensorial.

Se seleccionó y entrenó un panel de 15 jueces sensoriales (10 mujeres y 5 varones) quienes fueron estudiantes de la Escuela de Ciencia de los Alimentos de la Universidad Nacional Mayor de San Marcos, entrenados según normas NTP-ISO (NTP-ISO, 8586-1 ${ }^{18}$, NTP-ISO 8586$2{ }^{19} \mathrm{y}$ NTP - ISO $6658^{20}$ ), en las propiedades sensoriales y escalas a utilizar. Los jueces entrenados evaluaron los atributos consistencia y sabor en los tres tratamientos (TP01, TP02 y TP03).

En la evaluación sensorial del atributo consistencia se utilizó una escala no estructurada de $10 \mathrm{~cm}$, tomando como referencia en los extremos dos anclas $(0=$ agua, $10=$ leche condensada). El atributo sabor fue evaluado utilizando la prueba de ordenamiento $(1=$ sabor muy agradable y 3 = sabor menos agradable).

La prueba se realizó en un ambiente iluminado con luz blanca. Las bebidas fueron servidas en vasos descartables de tres onzas acompañados de un vaso de agua de mayor capacidad (5 onzas), las muestras fueron codificadas con números aleatorios de tres dígitos y presentadas en un diseño de bloques completamente al azar. El panel fue instruido para no ingerir alimentos, masticar chicle ni fumar por lo menos una hora antes de la realización de las pruebas, para no influir en los resultados. Se analizó cada atributo en una sesión separada utilizando una ficha de evaluación, que permitió que los participantes hicieran comentarios con respecto a los atributos evaluados.

\section{Prueba de nivel de agrado de consumidores}

La evaluación del consumidor es una experiencia caracterizada por una actitud ${ }^{4}$, en este caso el nivel de agrado o desagrado de la bebida. La evaluación del nivel de agrado de las bebidas elaboradas (tres tratamientos) se realizó con un panel de 50 consumidores ( 23 mujeres y 27 varones) de edades entre 16 y 40 años que consumen tradicionalmente la bebida y que residen en diferentes distritos de Lima. Se evaluaron las muestras empleando una escala hedónica de 5 puntos, desde 1 indicando alto nivel de agrado, 5 que indica mucha aversión y 3 indiferencia.

Las muestras fueron servidas a temperatura ambiente, en vasos descartables codificados con números aleatorios de tres dígitos, además se presentó agua mineral para beber entre muestras.

\section{Análisis estadístico}

Los resultados de las características físico-químicas de las muestras de bebida (tres tratamientos térmicos diferentes): $\mathrm{pH}$, grados Brix, viscosidad fueron evaluados por análisis de varianza.

Para el análisis sensorial con el panel de jueces expertos, se analizaron un total de 12 muestras de la bebida de quinua y piña ( 3 tratamientos $\times 2$ repeticiones $\times 2$ muestras de cada tratamiento) por panelista. Se realizó el análisis de varianza de dos vías (ANOVA) para la variable consistencia de la bebida, posteriormente se compararon las medias mediante la prueba de Tukey. El atributo sabor evaluado por el panel de jueces expertos y el nivel de agrado del consumidor se evaluaron con la prueba estadística no paramétrica de Friedman. Todas las pruebas se realizaron con un nivel de significancia del $95 \%$.

El modelo estadístico utilizado en este estudio fue: Yij $=\mu+F i+E E$. Donde Yij representa la respuesta del consumidor $j$ que recibe la bebida $i, \mu$ es el promedio del grado de aceptación, Fi representa el efecto del tratamiento y EE es el error experimental que representa la variabilidad entre las respuestas de los consumidores dentro de los tratamientos. Se empleó el software estadístico InfoStat versión estudiantil.

\section{RESULTADOS:}

\section{Determinación de los tratamientos térmicos para la elaboración de la bebida}

Considerando al Bysosschlamys fulva como microorganismo de referencia, se calcularon los tiempos correspondientes a los tratamientos de pasteurización a las temperaturas: $85^{\circ} \mathrm{C}, 90^{\circ} \mathrm{C}$ y $95^{\circ} \mathrm{C}$.

Tabla 1. Parámetros para el tratamiento térmico de la bebida de
piña y quinua
\begin{tabular}{ccc} 
Tratamiento Térmico & Temperatura $\left({ }^{\circ} \mathrm{C}\right)$ & Tiempo (minutos) \\
\hline TP01 & 85 & 26 \\
TP02 & 90 & 7 \\
TP03 & 95 & 2 \\
\hline
\end{tabular}

\section{Evaluación fisicoquímica}

La Tabla 2 muestra los valores medios de las propiedades fisicoquímicas cuantificadas por triplicado para las muestras de bebida de piña y quinua elaboradas en tres tratamientos. No hubo diferencias estadísticamente significativas $(\mathrm{p}>0,05)$ en todos los valores de $\mathrm{pH}$ y ${ }^{\circ}$ Brix. La viscosidad presenta diferencias estadísticamente sig- 
nificativas entre el tratamiento TP03 comparado con el TP01 y TP02.

Tabla 2. Características físico químicas de la bebida por tratamientos

\begin{tabular}{cccc}
\hline Tratamiento & ${ }^{\circ}$ Brix & pH & Viscosidad (cp) \\
\hline TP01 & $16^{\mathrm{a}}$ & $3,89^{\mathrm{a}}$ & $68,7^{\mathrm{a}}$ \\
TP02 & $16^{\mathrm{a}}$ & $3,89^{\mathrm{a}}$ & $66,1^{\mathrm{a}}$ \\
TP03 & $16^{\mathrm{a}}$ & $3,88^{\mathrm{a}}$ & $62,0^{\mathrm{b}}$ \\
\hline
\end{tabular}

Medias con una letra común no son significativamente diferentes ( $p>0.05$ ). Los datos son valores medios de tres repeticiones

\section{Análisis sensorial de la bebida de quinua y pińa}

Se aplicó el análisis de varianza para las calificaciones del atributo sensorial consistencia para los tres tratamientos térmicos de la bebida de pińa y quinua, se realizó la prueba de comparaciones múltiples de Tukey por presentar diferencias estadísticamente significativas, el tratamiento TP01 fue estadísticamente diferente a los demás tratamientos en cuanto a la consistencia.

En la Tabla 3 se muestra la media y la desviación estándar de las calificaciones de asignadas por el panel de jueces entrenados. Además, se observa que el tratamiento TP01 en promedio fue calificado como el de mayor consistencia por los consumidores, los tratamientos TP02 y TP03 han obtenido similar calificación.

Tabla 3. Valores medios del atributo sensorial consistencia

\begin{tabular}{ccc}
\hline Tratamiento & Consistencia & $\mathbf{n}$ \\
\hline TP01 & $5,57^{\mathrm{a}} \pm 1,741$ & 15 \\
TP02 & $4,13^{\mathrm{b}} \pm 1,552$ & 15 \\
TP03 & $3,87^{\mathrm{b}} \pm 1,846$ & 15 \\
\hline
\end{tabular}

Los datos son valores medios de las calificaciones del atributo sensoria consistencia de los tres tratamientos de la bebida de piña y quinua de 15 panelistas entrenados $\pm S D$. Medias con una letra común no son significativamente diferentes $(p>0.05)$.

En la Tabla 4 se muestran las calificaciones medias, desviación estándar para el atributo sabor del panel de jueces entrenados ( 15 jueces) y para la calificación de nivel de agrado de los 50 consumidores. Los resultados de la prueba de Friedman evidencian que los tres tratamientos no son diferentes estadísticamente para el atributo sabor ni para el nivel de agrado de los consumidores.

Tabla 4. Valores medios para las puntuaciones de sabor y nivel de agrado del consumidor de los tres tratamientos de la bebida de piña y quinua

\begin{tabular}{ccc}
\hline Tratamiento & $\begin{array}{c}\text { Calificación del Sabor } \\
\text { (Panel entrenado) }\end{array}$ & $\begin{array}{c}\text { Nivel de agrado del } \\
\text { consumidor }\end{array}$ \\
\hline TP01 & $2,20^{\mathrm{a}} \pm 0,941$ & $2,02^{\mathrm{a}} \pm 0,98$ \\
TP02 & $1,73^{\mathrm{a}} \pm 0,704$ & $2,16^{\mathrm{a}} \pm 1,11$ \\
TP03 & $2,07^{\mathrm{a}} \pm 0,799$ & $2,18^{\mathrm{a}} \pm 1,22$ \\
\hline
\end{tabular}

Valores promedio \pm SD obtenidos mediante el análisis sensorial de sabor (15 jueces entrenados) y nivel de agrado del consumidor (50 consumidores no entrenados) para los tres tratamientos de la bebida de piña y quinua. Medias con una letra común no son significativamente diferentes $(p>0,05)$.

En la Fig. 1 se muestran los resultados de las calificaciones promedio del nivel de agrado del consumidor por género, donde no existen diferencias significativas entre las calificaciones de nivel de agrado del consumidor por género con un nivel de significancia del $95 \%$.

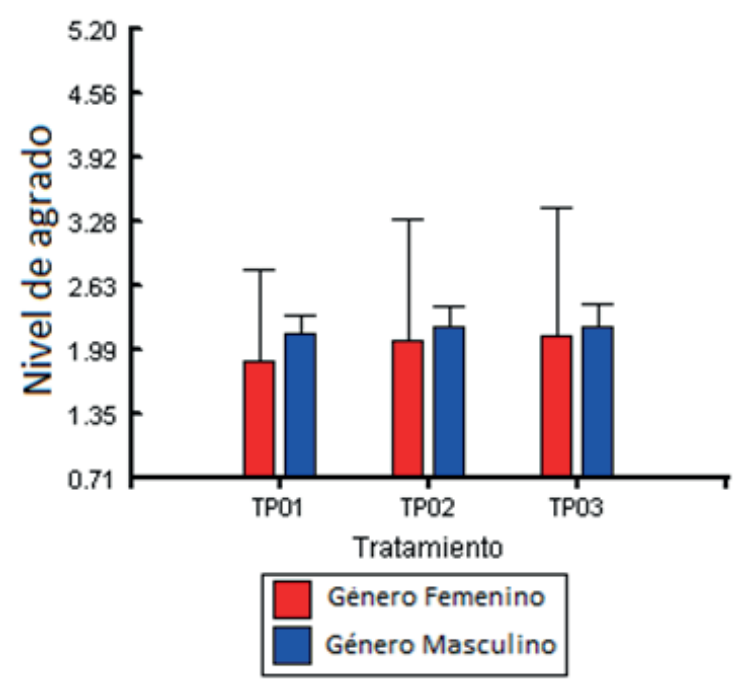

Figura 1. Calificación promedio del nivel de agrado consumidor por género

La Figura 2 muestra la calificación promedio de cada uno de los tres tratamientos de la bebida según el rango de edad (16 a 18, 19 a 22 y 22 a 40). Se aplicó el análisis de varianza para las respuestas de nivel de agrado en función al rango de edades y la prueba de comparaciones múltiples de Tukey con un nivel de significancia del 95 $\%$, por lo que se puede afirmar que la muestra TP02 es mejor calificada para el rango de edades entre 16 a 18 ańos en cuanto al nivel de agrado y el grupo de edades entre 23 a 40 años calificó a las bebidas en el nivel de agrado "indiferente". La muestra TP03 recibió la mejor calificación para el grupo de rango de edades entre 16 a 18 años y a su vez la calificación más baja del grupo de edades entre 23 a 40 ańos.

\section{DISCUSIÓN}

Las muestras de bebida fueron elaboradas con la misma formulación y tres tratamientos térmicos distintos (Tabla 1), presentaron valores iguales de ${ }^{\circ}$ Brix y $\mathrm{pH}$ (Tabla 2). El tratamiento térmico que empleó la menor temperatura y mayor tiempo (TP01), presentó mayor viscosidad ( $p>0.05$ ), estas diferencias sólo reflejan las modificaciones que se dan en función del tratamiento térmico, ya que la viscosidad está influenciada por la temperatura ${ }^{21}$.

Las diferencias en cuanto a la viscosidad en los tres tratamientos fueron percibidas por el panel de jueces entrenados en la calificación promedio de la consistencia sensorial (Tabla 3), sin embargo, esto no significó una menor calificación en el nivel de agrado del consumidor, contradiciendo la afirmación, que la evaluación de alimentos líquidos por el consumidor tiene relación con la viscosidad y consistencia del producto ${ }^{22}$. 


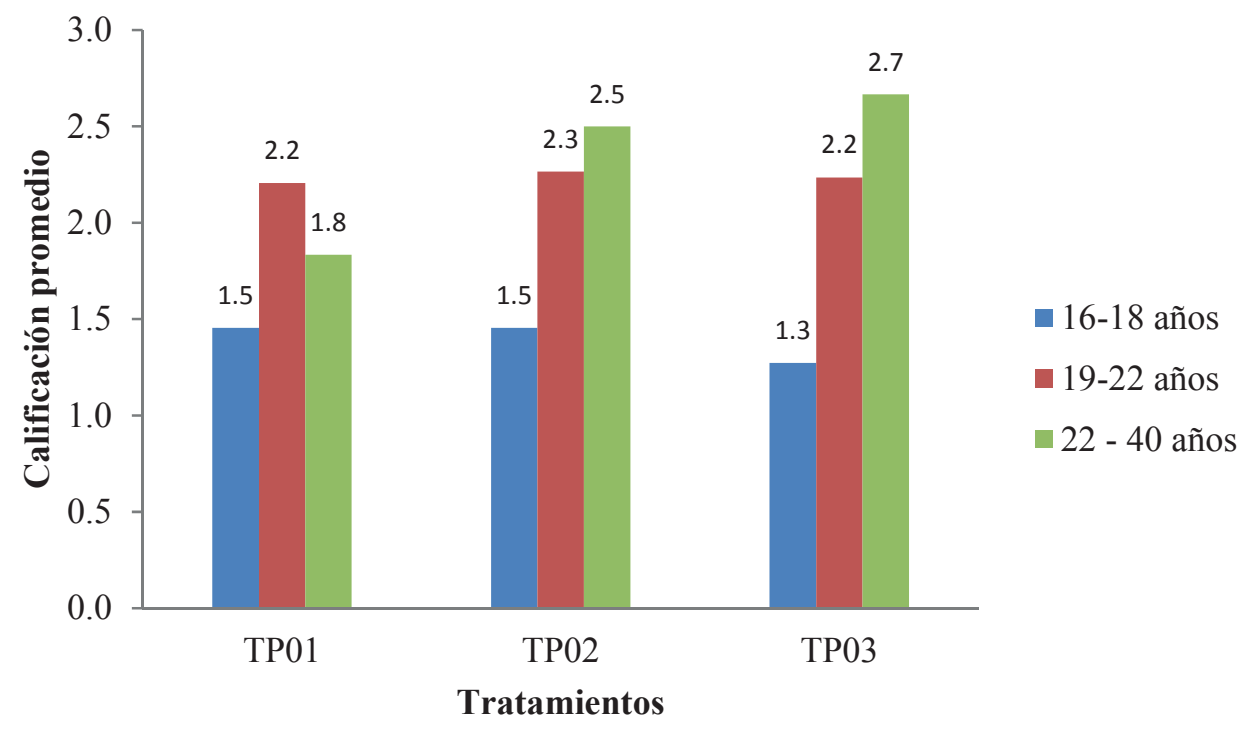

Figura 2. Calificación promedio por rango de edades

El gusto de los alimentos se puede detectar gracias a los receptores de la lengua que se encuentran en las papilas gustativas, siendo el sabor la sensación compleja integrada por la percepción simultanea del gusto y olor a través de la boca ${ }^{23,24}$. Las frutas tropicales como la piña ofrecen al consumidor una agradable experiencia sensorial, beneficios para la salud, al igual que la quinua. Sin embargo a pesar de las propiedades saludables de un alimento, los consumidores no comprometen el sabor de los alimentos a cambio de la salud ${ }^{3}$; el sabor tiene una conexión directa con la memoria, por lo que tiene vinculaciones emocionales que quedan en la mente y en el corazón de quien las consume ${ }^{24}$, es decir, el consumidor toma la actitud de aceptación o rechazo de un alimento en función a las emociones que relaciona con un sabor determinado, es probable que el sabor sea de importancia sustancial para determinar si los alimentos son aceptados o no ${ }^{25}$. El sabor pobre tiene un impacto negativo en la aceptación sensorial, por tanto, fue importante evaluar el sabor de las muestras de los tres tratamientos de la bebida evaluada, obteniéndose que el sabor tuvo una correlación positiva con la calificación del nivel de agrado (Tabla 4). Actualmente, el consumidor valora mucho el sabor de los alimentos y que estos contribuyan a un estilo de vida saludable, por lo que la industria alimentaria ha desarrollado productos que no se caracterizan por precios, sino por su calidad ${ }^{26}$.

La Tabla 4 muestra la calificación promedio del nivel de agrado de la bebida por tratamientos, estos no presentaron diferencias significativas ( $p>0,05)$, la Figura 1, presenta el gráfico de barras de la calificación promedio de la nivel de agrado por géneros, observándose que el género femenino calificó mejor en promedio el nivel de agrado de la bebida, sin embargo esta puntuación presenta una mayor desviación estándar, lo que podría explicarse porque las mujeres están más relacionadas con el denominado "gusto por necesidad" que valoriza la estética, cuya búsqueda está cargada de emociones y nuevas experiencias, dedicando tiempo y recursos a su bienestar; lo cual despliega toda una simbología de la prevención en pro de una mejor calidad de vida ${ }^{24}$.

Respecto a la relación del nivel de agrado de los consumidores por rangos de edad (Figura 2), los consumidores más jóvenes son los que dan las mejores calificaciones por tratamiento.

\section{CONCLUSIONES}

El tratamiento térmico afectó la viscosidad y la consistencia de los tres tratamientos, la consistencia no fue determinante en la respuesta del consumidor, pudiendo existir correlación positiva en la preferencia del consumidor con el sabor. La bebida elaborada gusta mucho al consumidor, siendo especialmente preferida por el consumidor de género femenino y los consumidores más jóvenes (16 - 18 años de edad).

\section{REFERENCIAS BIBLIOGRÁFICAS}

1. Higuchi A. Características de los consumidores de productos orgánicos y expansión de su oferta en Lima. Apuntes 2015; 42 (77): 57 - 89.

2. Verbeke W. Consumer acceptance of functional foods: sociodemographic, cognitive and attitudinal determinants. Food Qual Prefer. 2005; 16: 45-57.

3. Ramirez-Navas J, Callejas L, Quiceno C, Valencia Y. Consumer preference and acceptance of two Colombian sweet miIks: results of a consumer survey. UGCiencia 2015; 21: 9-15.

4. Huapaya E, Salazar J, Vera R. Calidad nutricional de desayunos ofrecidos en puesto de venta ambulatoria y nivel de conocimientos sobre alimentación de sus vendedores en el cercado de Lima. Renut 2011; 5 (15): 788- 796. 
5. Burbano J. Influencia de la pasteurización abierta y al vacío en las propiedades fisicoquímicas y el nivel de agrado de un néctar de piña (Ananas comosus L.), naranjilla (Solanum quitoense Lam.) y borojó (Borojoa patinoi Cuatrec.). [Trabajo de Graduación de la Carrera de Ingeniería en Alimentos]. Ecuador: Universidad Técnica de Ambato; 2015.

6. Blessing I, Offia-Oluan O, Ekwunife. Production and evaluation of the physico-chemical and sensory qualities of mixed fruit leather and cakes produced from apple (Musa pumila), banana (Musa sapientum), pineapple(Ananas comosus). Nigerian Food J. 2015; 33: 22-28

7. Domínguez C. Formulación y pasterización de una bebida con mezclas de jugos no clarificados de piña-guayaba-mango. [Tesis profesional Maestría en Ciencia de Alimentos]. Puebla - México: Universidad de las Américas Puebla; 2004.

8. Bhargava A, Shukla S, Ohri D. Chenopodium quinoa - An Indian perspective. Ind Crops Prod. 2006; 23(1):73 -87.

9. Nongonierma A, Le Maux S, Dubrulle C, Barre C, FitzGerald R. Quinoa (Chenopodium quinoa Willd.) protein hydrolysates with in vitro dipeptidyl peptidase IV (DPP-IV) inhibitory and antioxidant properties. J Cereal Sci. 2015; 65 (1): 112-118

10. Gonzalez M, Wells G, Fischer S, Escuredo O. Chemical characteristics and mineral composition of quinoa by near-infrared spectroscopy. J Sci Food Agric. 2014; 94: 876 -881.

11. Dini I, Tenore G, Dini A. Antioxidant compound contents and antioxidant activity before and after cooking in sweet and bitter Chenopodium quinoa seeds. LWT - Food Sci Technol. 2010; 43: 447-451.

12. Jay J, Loessner M, Golden D. Microbiología moderna de los alimentos. $5^{a}$ ed. Editorial Acribia S.A.; 2009.

13. Encina C, Bernal A, Rojas D. Efecto de la temperatura de pasteurización y proporción de mezclas binarias de pulpa de carambola y mango sobre su capacidad antioxidante lipofílica. Rev Ing Industrial 2013; 31: 197-219.

14. Malaga R, Guevara A, Araujo M. Efecto del Procesamiento de puré de Aguaymanto (Physalis peruviana L.), sobre los compuestos bioactivos y la capacidad antioxidante. Rev Soc Quím Perú, 2013; 79 (2): 162-174.
15. Villareal Y, Fernado D, Osorio O, Felipe A. Efecto de pasteurización sobre características sensoriales y contenido de vitamina $C$ en jugos de frutas. Biotecnología en el Sector Agropecuario y Agroindustrial. 2013; 11(2): 66-75.

16. Monteiro M, Lara Dos Santos V, Trindade M. Consumers' perception of beef burgers with different healthy Attributes. LWT - Food Sci Technol. 2014; 59: 1227-1232.

17. INDECOPI. Análisis sensorial. Vocabulario. NTP-ISO 5492. Lima: INDECOPI, 2008.

18. INDECOPI. Análisis Sensorial. Guía general para selección, entrenamiento y control de jueces. Parte 1: Catadores. NTP 8586-1. Lima: INDECOPI, 2008

19. INDECOPI. Análisis sensorial. Guía general para la selección, entrenamiento y control de jueces. Parte 2: Expertos. NTP 8586-2. Lima: INDECOPI, 2008

20. INDECOPI. Análisis Sensorial. Metodología, Lineamientos Generales. NTP 6658. Lima: INDECOPI, 2008

21. Nayik G, Dar B, Nanda V. Rheological behavior of high altitude Indian honey varieties as affected by temperature. J Saudi Soc of Agric Sci. 2018; 17: 323-329.

22. Badui, S. Química de los Alimentos. $5^{\text {a }}$ edición. Editorial Pearson; 2013.

23. Sancho J, Bota E y De Castro J. Introducción al análisis sensorial de los alimentos. México; Editorial Alfaomega; 2002.

24. Garzón C. Significados sociales y culturales que median en la experiencia multisensorial del sabor con respecto al consumo de marcas de alimentos. Rev GEON. 2015; (4): 7 - 13.

25. Schouteten J, De Steur H, De Pelsmaeker S, Lagast S, Juvinal J, De Bourdeaudhuij I, et al. Emotional and sensory profiling of insect-, plant- and meat-based burgers under blind, expected and informed conditions. Food Qual Prefer. 2016; 52: 27-31.

26. Mărcută L, Mărcută A, Mârza B. Modern Tendencies in Changing the Consumers' Preference. In: $21^{\text {st }}$ International Economic Conference Sibiu, Romania. 2014. Procedia Econ Finance. 2014; 16: 535 - 539 .

Conflicto de intereses: Los autores declaran no tener conflictos de interés.

Fuente de financiamiento: Autofinanciado 